\title{
Special Issue on Semantic Technology
}

\author{
Ryutaro Ichise $^{1} \cdot$ Stephen Muggleton ${ }^{2} \cdot$ Kouji Kozaki $^{3} \cdot$ Freddy Lecue $^{4}$. \\ Dongyan Zhao ${ }^{5} \cdot$ Takahiro Kawamura $^{6}$
}

Published online: 7 November 2019

(c) Ohmsha, Ltd. and Springer Japan KK, part of Springer Nature 2019

It is our great pleasure to publish the special issue on semantic technology. The purpose of this special issue is to bring together researchers in the Semantic Technology research community and other areas of semantic-related technologies to present their innovative research results or novel applications of semantic technologies. In this special issue, we solicit papers on various aspects of semantic technology from various fields such as ontology, semantic web, and novel applications of semantic technologies to promote research activities in the fields.

Nineteen papers were submitted. All papers were referred in accordance with the usual rigorous standards of New Generation Computing. After several interactions with the authors, we accepted nine papers for this special issue. The topics covered mainly the novel applications of semantic technologies. In particular, many papers are related to ontologies. It shows the current trend in Semantic Technologies.

We would like to express sincere thanks to the authors for their contributions and the anonymous referees for reviewing the papers in a short period of time.

\section{Guest Editor-in-Chief}

Ryutaro Ichise

Ryutaro Ichise

ichise@nii.ac.jp

1 National Institute of Informatics, 2-1-2, Hitotsubashi, Chiyoda-ku, Tokyo 101-8430, Japan

2 Imperial College London, 180 Queen's Gate, London SW7 2BZ, UK

3 Osaka Electro-Communication University, 18-8 Hatsucho, Neyagawa-shi, Osaka 572-8530, Japan

4 Inria Sophia Antipolis-Méditerranée, 2004, route des Lucioles, BP 93, 06902 Sophia Antipolis Cedex, France

5 Peking University, No.128 North Zhongguancun Street, Haidian District, Beijing 100080, China

6 National Agriculture and Food Research Organization, 3-1-1 Kannondai, Tsukuba, Ibaraki 305-8517, Japan 


\section{Board Members}

Stephen Muggleton

Kouji Kozaki

Freddy Lecue

Dongyan Zhao

Takahiro Kawamura

Publisher's Note Springer Nature remains neutral with regard to jurisdictional claims in published maps and institutional affiliations. 